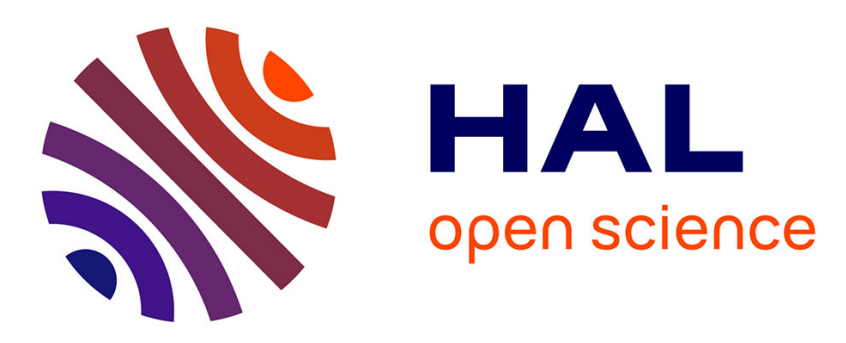

\title{
Application of conditioned structure functions to exploring influence of premixed combustion on two-point turbulence statistics
}

Vladimir Sabelnikov, Andrei N. Lipatnikov, Shinnosuke Nishiki, Tatsuya

Hasegawa

\section{To cite this version:}

Vladimir Sabelnikov, Andrei N. Lipatnikov, Shinnosuke Nishiki, Tatsuya Hasegawa. Application of conditioned structure functions to exploring influence of premixed combustion on twopoint turbulence statistics. Proceedings of the Combustion Institute, 2019, 37 (2), pp.2433-2441. 10.1016/j.proci.2018.08.029 . hal-02315612

\section{HAL Id: hal-02315612 \\ https://hal.science/hal-02315612}

Submitted on 14 Oct 2019

HAL is a multi-disciplinary open access archive for the deposit and dissemination of scientific research documents, whether they are published or not. The documents may come from teaching and research institutions in France or abroad, or from public or private research centers.
L'archive ouverte pluridisciplinaire HAL, est destinée au dépôt et à la diffusion de documents scientifiques de niveau recherche, publiés ou non, émanant des établissements d'enseignement et de recherche français ou étrangers, des laboratoires publics ou privés. 


\section{Application of conditioned structure functions to exploring influence of premixed combustion on two-point turbulence statistics}

Vladimir A. Sabelnikov ${ }^{1,2}$, Andrei N. Lipatnikov ${ }^{3}$, Shinnosuke Nishiki ${ }^{4}$, Tatsuya Hasegawa ${ }^{5}$

${ }^{1}$ ONERA - The French Aerospace Laboratory, F-91761 Palaiseau, France

${ }^{2}$ Central Aerohydrodynamic Institute (TsAGI), 140180 Zhukovsky, Moscow Region, Russian Federation

${ }^{3}$ Department of Mechanics and Maritime Sciences, Chalmers University of Technology, Gothenburg, 41296 Sweden

${ }^{4}$ Department of Mechanical Engineering, Kagoshima University, Kagoshima 890-0065, Japan,

${ }^{5}$ Institute of Materials and Systems for Sustainability, Nagoya University, Nagoya 464-8603, Japan

Corresponding author: Prof. Vladimir Sabelnikov, ONERA - The French Aerospace Laboratory, F-91761 Palaiseau,

France, vladimir.sabelnikov@ onera.fr

Colloquium: Turbulent flames

Total length: 6181 words

- Main text: 3918 words

- Equations: $(8$ lines +8 blank lines $) \times(7.6$ words/line $)=122$ words

- References: $(53+2) \times(2.3$ lines/reference $) \times(7.6$ words/line $)=961$ words

- Figure 1: $(154 \mathrm{~mm}+10 \mathrm{~mm}) \times(2.2$ words $/ \mathrm{mm})+109$ words $=470$ words

- Figure 2: $(47 \mathrm{~mm}+10 \mathrm{~mm}) \times(2.2$ words $/ \mathrm{mm})+14$ words $=139$ words

- Figure 3: $(101 \mathrm{~mm}+10 \mathrm{~mm}) \times(2.2$ words $/ \mathrm{mm})+55$ words $=297$ words

- Figure 4: $(101 \mathrm{~mm}+10 \mathrm{~mm}) \times(2.2$ words $/ \mathrm{mm})+28$ words $=272$ words

We agree to pay charges for color publications if color printing is deemed necessary. 


\begin{abstract}
In order to investigate the influence of combustion-induced thermal expansion on turbulent flow within a premixed flame brush, a new method is introduced. The method consists in analyzing structure functions of the velocity field, which characterize velocity difference in two points $\mathrm{A}$ and $\mathrm{B}$, with the structure functions being conditioned to various events; (i) unburned reactants in both points, (ii) combustion products in both points, (iii) intermediate states of the mixture in both points, (iv) the reactants in one point and the products in another point, (v) the reactants in one point and an intermediate state in another point, and (vi) the products in one point and an intermediate state in another point. Such structure functions and relevant probabilities are defined in the paper. Subsequently, the structure functions and the probabilities are extracted from Direct Numerical Simulation (DNS) data obtained from two statistically planar, 1D, fully-developed, weakly turbulent, premixed flames characterized by two significantly different (7.52 and 2.50 ) density ratios, with all other things being approximately equal. Obtained results indicate that (i) the conditioned structure functions differ significantly from the mean structure functions and (ii) the newly introduced approach could convey information important for understanding fundamentals of flame-turbulence interaction and finding issues that require further research. In particular, application of the approach to the aforementioned DNS data shows that the combustion-induced thermal expansion substantially affects smallscale two-point velocity statistics in the incoming constant-density turbulent flow of unburned reactants within a premixed
\end{abstract}

flame brush.

Keywords: premixed combustion, turbulent flames, flame-generated turbulence, thermal expansion, DNS

\title{
1. Introduction
}

Since the seminal work by Karlovitz et al. [1] and Scurlock and Grover [2], the influence of combustion-induced thermal expansion on turbulent flow within a premixed flame brush was studied by various research groups, as reviewed elsewhere [3-6]. The majority of such studies addressed the problem of flame-generated turbulence [1,2] and their focus was placed on the behavior of the rms turbulent velocity $u^{\prime}$ and Reynolds stresses within the mean flame brush, particularly, downstream of the instantaneous flame front. However, until recently, certain fundamentally important and challenging subjects such as (i) eventual influence of thermal expansion on turbulent flow of unburned reactants both within and upstream of a mean flame brush or (ii) behavior of two-point turbulence characteristics, which provide insights into energy distribution over length scales, in premixed flames were rarely addressed. For instance, over many decades of research into premixed combustion, only a few spectra of turbulence energy were obtained from flames by measuring temporal correlations [7-12], with significant influence of combustion on the spectra being documented in these experiments. Recent advancement in Direct Numerical Simulation (DNS) of turbulent reacting flows provided the combustion community with a powerful research tool capable for evaluating various characteristics of turbulent flames, including two-point turbulence statistics. Nevertheless, only a few studies of the influence of premixed combustion on turbulence-energy spectra have yet 
been performed by extracting two-point spatial correlations from DNS data [13-16]. As these studies revealed substantial changes of the spectra at small wavelengths comparable with the relevant laminar flame thicknesses, further research into behavior of two-point turbulence characteristics in premixed flames is definitely required, in particular, in order to examine whether or not (or under which conditions) the Kolmogorov theory may be used to characterize turbulence in flames.

In order to explore the issue more efficiently, the well-known, but sufficiently expensive spectral method is worth being complemented with other research tools, which either could be easier to use, or/and could be applicable under different conditions, or/and could offer an opportunity to take a look into the influence of combustion on turbulence from another side. Structure functions widely used by the turbulence community [17-21] appear to be such a tool. While higher order structure functions are particularly well known due to their importance for fundamental research into intermittency in turbulent flows $[18,19]$, even the second-order structure functions are widely used equally with spectral methods, with each of the two approaches having its advantages and disadvantages [21]. For instance, the latter structure functions were successfully used to develop models for large-eddy simulations of turbulent flows [22]. It is of interest to note that Kolmogorov developed his theory of the locally homogeneous, isotropic turbulence by analyzing the second-order structure functions [23], whereas the $5 / 3$ spectrum of turbulence in the inertial subrange was predicted by his $\mathrm{PhD}$ student Obukhov [24].

Contrary to the wide use of the structure functions by the turbulence community, this method has yet been beyond the focus of research into combustion. The present authors are aware on a single application of the structure functions to premixed flames, i.e., a theoretical study by Kuznetsov [25] of manifestations of the hydrodynamic instability [26] in turbulent flows. The goal of the present work is to fill this gap and to advance applications of the structure functions to examining the influence of combustion-induced thermal expansion on turbulence and, in particular, its two-point characteristics.

For this purpose, in the next section, the structure-function method is extended by introducing conditioned structure functions following an approach developed by Kuznetsov and Sabelnikov [18] to study intermittency in non-reacting turbulent flows. Subsequently, the newly extended method is applied to analyzing DNS data whose attributes are summarized in the third section. Obtained results are discussed in the fourth section followed by conclusions.

\section{Structure Functions}

The second-order structure functions of the velocity field are defined as follows [17-23]

$$
D_{i j}(\mathbf{r}, \mathbf{x}, t)=\left\langle\left[u_{i}(\mathbf{x}+\mathbf{r}, t)-u_{i}(\mathbf{x})\right]\left[u_{j}(\mathbf{x}+\mathbf{r}, t)-u_{j}(\mathbf{x})\right]\right\rangle
$$

where $\mathbf{x}$ and $\mathbf{x}+\mathbf{r}$ are two spatial points, $u_{i}$ is $i$-th component of the velocity vector $\mathbf{u}$, and $\langle q\rangle$ designates an ensemble averaged value of a quantity $q$. These structure functions characterize the second moment of the velocity difference across 
scales of size $r=|\mathbf{r}|$. Accordingly, they measure the energy of such and smaller eddies and, therefore, allow us to examine the energy distribution over the spatial scales directly in the physical space, contrary to energy spectra which deal with wavenumbers. Due to this feature, the second order structures are widely used both in theoretical and experimental studies of turbulent flows [17-23].

Within a premixed turbulent flame brush, local velocity is increased due to a decrease in the local density $\rho$, caused by combustion-induced thermal expansion. Often, such density changes are localized to thin zones and the local velocity normal to the zone jumps at the burned side of the zone when compared to the unburned side, with such effects being most pronounced in the flamelet regime of premixed turbulent combustion [4-6]. Because such a velocity jump is hardly associated with turbulence, the use of mean (ensemble-averaged) quantities for characterizing turbulence in premixed flames appears to be a flawed approach [5,6], at least in the flamelet combustion regime. To resolve the problem, flow characteristics conditioned to unburned (or burned) mixture are often considered instead of mean flow characteristics, as reviewed elsewhere [5]. However, as far as energy spectra obtained from spatial correlations computed in DNS are concerned, the present authors are aware on a single study of the conditioned spectra [14], with that study being restricted to the leading edges of mean flame brushes. The structure functions offer an opportunity to gain insight into the conditioned two-point statistics of turbulent velocity field.

Indeed, let us consider a statistically stationary, planar, 1D premixed turbulent flame that propagates from right to left along the $x$-direction in the coordinate framework attached to the flame brush. ${ }^{1}$ The considered flame and flow are statistically homogeneous and isotropic in any transverse plane $x=$ const and we will restrict ourselves to structure functions measured for two points $\mathbf{x}_{A}=\left\{x_{A B}, y_{A}, z_{A}\right\}$ and $\mathbf{x}_{B}=\left\{x_{A B}, y_{A}+r_{y}, z_{A}+r_{z}\right\}$ that belong to the same transverse plane $x=$ $x_{A B}$, i.e., $\mathbf{r}=\left\{0, r_{y}, r_{z}\right\}$

Let us introduce three indicator functions $I_{k}(\mathbf{x}, t)$ such that $I_{1}(\mathbf{x}, t)=1$ in reactants and vanishes otherwise, $I_{2}(\mathbf{x}, t)=$ 1 in products and vanishes otherwise, whereas $I_{3}(\mathbf{x}, t)$ vanishes in the reactants and products, but is equal to unity otherwise. Obviously, $I_{1}(\mathbf{x}, t)+I_{2}(\mathbf{x}, t)+I_{3}(\mathbf{x}, t)=1$. Then, for any quantity $q$, the following identity holds

$$
\langle q\rangle=\left\langle q \sum_{k=1}^{3} I_{k}\right\rangle=\sum_{k=1}^{3}\left\langle q I_{k}\right\rangle=\sum_{k=1}^{3} \mathrm{P}_{k}\langle q\rangle_{k},
$$

where $\langle q\rangle_{k} \equiv\left\langle q I_{k}\right\rangle / \mathrm{P}_{k}$ designates the value of $q$ conditioned to $k$-th state of the mixture and $\mathrm{P}_{k}=\left\langle I_{k}\right\rangle$ is the probability of finding this state. Henceforth, dependencies of various quantities on time $t$ and spatial coordinates $\mathbf{x}$ are not specified unless the opposite is required, whereas $q_{A}$ and $q_{B}$ designate values of a quantity $q$, measured in points $\mathbf{x}_{A}$ and $\mathbf{x}_{B}=\mathbf{x}_{A}+\mathbf{r}$, respectively, at the same instant $t$.

\footnotetext{
${ }^{1}$ The approach can be used in a general statistically inhomogeneous, unsteady case.
} 
By averaging the following identity

$$
\begin{gathered}
\left(u_{B, i}-u_{A, i}\right)\left(u_{B, j}-u_{A, j}\right) \\
=\left(u_{B, i}-u_{A, i}\right)\left(u_{B, j}-u_{A, j}\right)\left(\sum_{k=1}^{3} I_{B, k}\right)\left(\sum_{l=1}^{3} I_{A, l}\right)
\end{gathered}
$$

over transverse plains and time, we arrive at

$$
D_{i j}(x, r)=\sum_{k=1}^{3} \sum_{l=1}^{3} D_{i j, k l}(x, r) \mathrm{P}_{k l}(x, r),
$$

where subscripts $i$ and $j$ refer to spatial coordinates, subscripts $k$ and $l$ refer to the state of the mixture, $D_{i j, k l}(x, r) \equiv$ $\left\langle\left(u_{B, i}-u_{A, i}\right)\left(u_{B, j}-u_{A, j}\right) I_{B, k} I_{A, l}\right\rangle / \mathrm{P}_{k l}$ are conditioned structure functions, and $\mathrm{P}_{k l}(x, r)=\left\langle I_{B, k} I_{A, l}\right\rangle$ are the probabilities that the mixture states $k$ and $l$ are recorded in points $\mathbf{x}_{B}$ and $\mathbf{x}_{A}$, respectively, at the same instant. In Eq. (4), the tensor $D_{i j, k l}(x, r)$ depends solely on $x=|\mathbf{x}|$ and $r$, rather than $\mathbf{x}$ and $\mathbf{r}$, because the considered process is statistically homogeneous and isotropic in a transverse plane and $\mathbf{r}=\left\{0, r_{y}, r_{z}\right\}$. In such a case, there are six different conditioned structure-function tensors and six different probabilities; $D_{i j, 11}$ and $\mathrm{P}_{11}$ (reactants in both points), $D_{i j, 22}$ and $\mathrm{P}_{22}$ (products in both points), $D_{i j, 33}$ and $\mathrm{P}_{33}$ (intermediate states of the mixture in both points), $D_{i j, 12}=D_{i j, 21}$ and $\mathrm{P}_{12}=\mathrm{P}_{21}$ (reactants in one point, but products in another point), $D_{i j, 13}=D_{i j, 31}$ and $\mathrm{P}_{13}=\mathrm{P}_{31}$ (reactants in one point, but an intermediate state in another point), and $D_{i j, 23}=$ $D_{i j, 32}$ and $\mathrm{P}_{23}=\mathrm{P}_{32}$ (products in one point, but an intermediate state in another point).

\section{DNS Attributes}

In the rest of the present paper, we will illustrate certain opportunities associated with the newly introduced conditioned structure functions by extracting them from a DNS database created by Nishiki et al. [27,28] about 15 years ago. The choice of this database, which may appear to be outdated when compared to recent DNS data generated in the case of complex combustion chemistry and a high ratio of $u^{\prime}$ to the laminar flame speed $S_{L}$, e.g. [29-33], requires comments. The point is that the focus of the following discussion is placed on the influence of combustion-induced thermal expansion on the structure functions conditioned to either reactants or products. Therefore, detailed description of intermediate states of the mixture (e.g., complex combustion chemistry) appears to be of secondary importance when compared to two other major requirements. First, a study of any new approach should be started under conditions that are associated with the strongest manifestation of effects the approach aims at. Accordingly, to make the thermal expansion effects as strong as possible, heat release and density drop should be localized to sufficiently thin zones and the velocity jump across such zones should be sufficiently large when compared to $u^{\prime}$. In other words, the flamelet regime of premixed turbulent combustion associated with a low ratio of $u^{\prime} / S_{L}$ should be addressed. The DNS by Nishiki et al. [27,28] dealt with this regime, as discussed in details elsewhere [34], whereas the majority or recent advanced DNS studies attacked other combustion regimes. Second, to 
better explore the thermal expansion effects, data obtained at significantly different density ratios $\sigma=\rho_{1} / \rho_{2}$ are required and the DNS database by Nishiki et al. [27,28] satisfies this requirement, as cases of $\sigma=2.5$ and 7.53 were simulated, with all other things being roughly equal.

Because the DNS data were discussed in details elsewhere $[27,28]$ and were already used by various research groups [34-48], we will restrict ourselves to a brief summary of those compressible simulations. They dealt with statistically planar, 1D, equidiffusive, adiabatic flames modeled by unsteady 3D continuity, Navier-Stokes, and energy equations, supplemented with a transport equation for the mass fraction $Y$ of a deficient reactant and the ideal gas state equation. The Lewis and Prandtl numbers were equal to 1.0 and 0.7 , respectively. Combustion chemistry was reduced to a single reaction. Temperature-dependence of molecular transport coefficients was taken into account, e.g., $v=v_{1}\left(T / T_{1}\right)^{0.7}$.

The computational domain was a rectangular box $\Lambda_{x} \times \Lambda_{y} \times \Lambda_{z}$ with $\Lambda_{x}=8 \mathrm{~mm}, \Lambda_{y}=\Lambda_{z}=4 \mathrm{~mm}$, and was resolved using a uniform rectangular $(2 \Delta x=\Delta y=\Delta z)$ mesh of $512 \times 128 \times 128$ points. Homogeneous isotropic turbulence $\left(u^{\prime}=0.53 \mathrm{~m} / \mathrm{s}, L=3.5 \mathrm{~mm}\right.$, and the turbulent Reynolds number $\left.R e_{t}=u^{\prime} L / v_{1}=96[27,28]\right)$ was generated in a separate box and was injected into the computational domain through the left boundary $x=0$. In the computational domain, the turbulence decayed along the direction $x$ of the mean flow. The flow was periodic in $y$ and $z$ directions.

At $t=0$, a planar laminar flame was embedded into statistically the same turbulence assigned for the velocity field in the entire computational domain. Subsequently, the inflow velocity was increased twice, i.e., $U\left(0 \leq t<t_{I}\right)=S_{L}<$ $U\left(t_{I} \leq t<t_{I I}\right)<U\left(t_{I I} \leq t\right)=S_{t}$, in order to keep the flame in the computational domain till the end $t_{I I I}$ of the simulations. Here, $S_{t}$ is the turbulent flame speed.

Three cases H, M, and L characterized by High, Medium, and Low, respectively, density ratios were studied [27,28]. In the following, we will restrict ourselves to (i) case $\mathrm{H}$ characterized by the highest $\sigma=7.53, S_{L}=0.6 \mathrm{~m} / \mathrm{s}, \delta_{L}=0.217 \mathrm{~mm}$, $D a=18, K a=0.21, S_{t}=1.15 \mathrm{~m} / \mathrm{s}$, and (ii) case L characterized by the lowest $\sigma=2.5, S_{L}=0.416 \mathrm{~m} / \mathrm{s}, \delta_{L}=0.158 \mathrm{~mm}$, $D a=17.3, K a=0.30$, and $S_{t}=0.79 \mathrm{~m} / \mathrm{s}$. Here, $\delta_{L}=\left(T_{2}-T_{1}\right) / \max \{|\nabla T|\}$ is the laminar flame thickness, $D a=$ $\left(L / u^{\prime}\right) /\left(\delta_{L} / S_{L}\right)$ and $K a=\left(u^{\prime} / S_{L}\right)^{3 / 2}\left(L / \delta_{L}\right)^{-1 / 2}$ are the Damköhler and Karlovitz numbers, respectively, evaluated at the leading edges of the mean flame brushes. The two flames are well associated with the flamelet combustion regime, e.g., various Bray-Moss-Libby expressions hold in cases H and L, see figures 1-4 in Ref. [34].

The DNS data were processed as follows. Mean quantities $\bar{q}(x)$ were averaged over transverse $y z$-planes and over time (220 and 200 snapshots in cases $\mathrm{H}$ and $\mathrm{L}$, respectively, stored during a time interval of $t_{I I I}-t_{I I} \approx 1.5 L / u^{\prime} \approx 10 \mathrm{~ms}$ ). Subsequently, $x$-dependencies were mapped to $\bar{c}$-dependencies using the spatial profiles of the Reynolds-averaged combustion progress variable $\bar{c}=1-\bar{Y} / Y_{1}$. The probabilities $\mathrm{P}_{k l}(r, \bar{c})$ were directly extracted from the DNS data, with reactants and products being associated with $c(\mathbf{x}, t)<\epsilon \ll 1$ and $c(\mathbf{x}, t)>1-\epsilon$, respectively. Variations in $\epsilon$ from 0.005 to 0.05 weakly affected results reported in the following, with a single exception of distance $r_{0}$ associated with a jump in $D_{i j, 12}$ 
from zero at $r<r_{0}$ to a finite value at $r>r_{0}$. As will be discussed later, $r_{0}$ is approximately equal to the distance between iso-surfaces of $c=\epsilon$ and $c=1-\epsilon$ in the laminar flame and, consequently, is decreased with increasing $\epsilon$. Results reported in the next section were computed using $\epsilon=0.05$.

The transverse structure functions $D_{x x, T}(r, \bar{c})$ for the axial velocity and their conditioned components were obtained by (conditionally) averaging $\left(u_{B}-u_{A}\right)^{2}$ over time and two sets of points; (i) $\mathbf{x}_{A}=\{x, y, z\}, \mathbf{x}_{B}=\{x, y+r, z\}$ and (ii) $\mathbf{x}_{A}=$ $\{x, y, z\}, \mathbf{x}_{B}=\{x, y, z+r\}$. Here, $\mathbf{u}=\{u, v, w\}, 0 \leq y<\Lambda_{y}, 0 \leq z<\Lambda_{z}$, and $0 \leq r<\Lambda_{y} / 2=\Lambda_{z} / 2$. The transverse mean (conditioned) structure functions $D_{y y, T}$ and $D_{z z, T}$ for the transverse velocities were obtained by (conditionally) averaging (i) $\left(v_{B}-v_{A}\right)^{2}$ and (ii) $\left(w_{B}-w_{A}\right)^{2}$, respectively, over time and (i) $\mathbf{x}_{A}=\{x, y, z\}, \mathbf{x}_{B}=\{x, y, z+r\}$ and (ii) $\mathbf{x}_{A}=\{x, y, z\}$, $\mathbf{x}_{B}=\{x, y+r, z\}$, respectively. On the contrary, the longitudinal mean (conditioned) structure functions $D_{y y, L}$ and $D_{z z, L}$ for the transverse velocities components were obtained by (conditionally) averaging (i) $\left(v_{B}-v_{A}\right)^{2}$ and (ii) $\left(w_{B}-w_{A}\right)^{2}$, respectively, over time and (i) $\mathbf{x}_{A}=\{x, y, z\}, \mathbf{x}_{B}=\{x, y+r, z\}$ and (ii) $\mathbf{x}_{A}=\{x, y, z\}, \mathbf{x}_{B}=\{x, y, z+r\}$, respectively. Finally, transverse and longitudinal structure functions for the transverse velocity were found as follows: $D_{y z, T}(r, \bar{c})=$ $0.5\left(D_{y y, T}+D_{z z, T}\right)$ and $D_{y z, L}(r, \bar{c})=0.5\left(D_{y y, L}+D_{z z, L}\right)$, respectively.

\section{Results and Discussion}

Typical mean (dots) and conditioned (lines) structure functions are shown in Fig. 1. These data were computed in the middle $(\bar{c}=0.50)$ of the H-flame brush in order for the probabilities $\mathrm{P}_{k l}(r, \bar{c})$ to be significant, see Fig. 2, thus, allowing us to obtain solid statistics. It is worth noting that the majority of mean or conditioned structure-function variances are slightly larger than the corresponding mean or conditioned structure functions, respectively, with the difference being more pronounced for the mean structure functions. However, the transverse structure function $D_{x x, T, 12}$ for the axial velocity $u$ and the longitudinal structure function $D_{y z, L, 12}$ for the transverse velocity $v$ or $w$, conditioned to unburned reactants in one point and burned products in another point, see solid lines marked with ub (i.e., unburned/burned) in Figs. 1a and 1c, respectively, are much larger than their variance. The focus of the subsequent discussion is placed on the mean structure functions shown in dots and structure functions conditioned to unburned reactants in both points (uu), unburned reactants and burned products (ub), and burned products in both points (bb), shown in bold lines (dashed, solid, and dotted-dashed lines, respectively). The following trends are worth emphasizing.

First, the mean structure functions, see dots, differ substantially from the conditioned structure functions, see lines, thus, showing that the velocity jumps associated with the unburned-burned intermittency strongly change two-point statistics of the velocity field within a premixed turbulent flame brush. Therefore, results plotted in Fig. 1 call for investigation of the conditioned fields in order to properly characterize the aforementioned two-point statistics. 

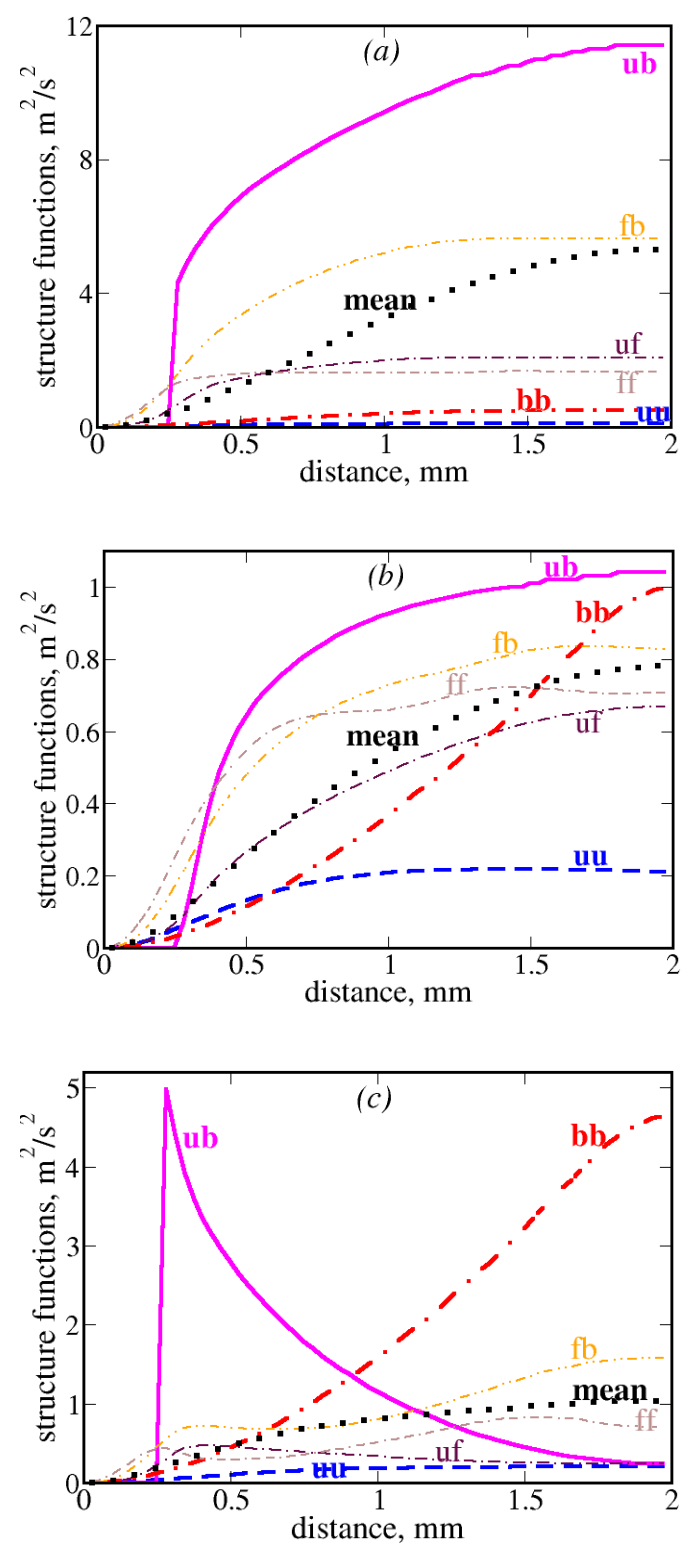

Fig. 1. Conditioned (color lines) and mean (black dots) structure functions obtained at $\bar{c}=0.50$ in case H. (a) Transverse structure functions $D_{x x, T}(r, \bar{c}=0.50)$ for axial velocity. (b) Transverse structure functions $D_{y z, T}(r, \bar{c}=0.50)$ for transverse velocities. (c) Longitudinal structure functions $D_{y z, L}(r, \bar{c}=0.50)$ for transverse velocities. The structure functions are conditioned to the following events; unburned mixture in both points (blue bold dashed lines marked with uu), unburned and burned mixtures (magenta bold solid lines, ub), unburned mixture and flamelet (maroon fine dotted-dashed lines, uf), burned mixture in both points (red bold dotted-dashed lines, bb), burned mixture and flamelet (orange fine double-dotted-dashed lines, fb), and flamelet in both points (brown fine double-dashed-dotted lines, ff).

Second, the unburned-burned structure functions $D_{x x, T, 12}, D_{y z, T, 12}$, and $D_{y z, L, 12}$ and the relevant probability $\mathrm{P}_{12}$ (solid lines in Figs. 1 and 2, respectively) vanish at $r<r_{0}=0.28 \mathrm{~mm}$, with this distance being equal to the distance between 
planes $c=\epsilon=0.05$ and $c=1-\epsilon=0.95$ in the unperturbed laminar flame $\mathrm{H}$. If $r>r_{0}$, then, all three structure functions are positive. Therefore, there are pairs of points $\left(\mathbf{x}_{A}, \mathbf{x}_{B}\right)$ such that (i) both points lie on a line parallel to a transverse coordinate axis and, hence, normal to another transverse axis, (ii) distance $r$ between the two points is close to $r_{0}$, and (iii) $c\left(\mathbf{x}_{A}, t\right)<0.05$ and $c\left(\mathbf{x}_{B}, t\right)>0.95$ or vice versa. However, the probability of such events is low if $r$ is close to $r_{0}$, see solid line in Fig. 2. Since, under conditions of the present DNS and at $\bar{c}=0.5$, the flamelet scalar structure is weakly perturbed when compared to the laminar flame [34], items (i)-(iii) considered together imply that the flamelet is locally parallel to the $x$-axis, i.e., the behavior of the conditioned structure functions at $r \approx r_{0}$ is controlled by flamelets characterized by $\left|n_{x}\right| \ll 1$ and either $\left|n_{y}\right| \ll 1$ or $\left|n_{z}\right| \ll 1$. Indeed, if the magnitude of $\left|n_{x}\right|$ be substantial, the $y$ or $z$-distance between two points characterized by $c\left(\mathbf{x}_{A}, t\right)<0.05$ and $c\left(\mathbf{x}_{B}, t\right)>0.95$ will be substantially larger than $r_{0}$.

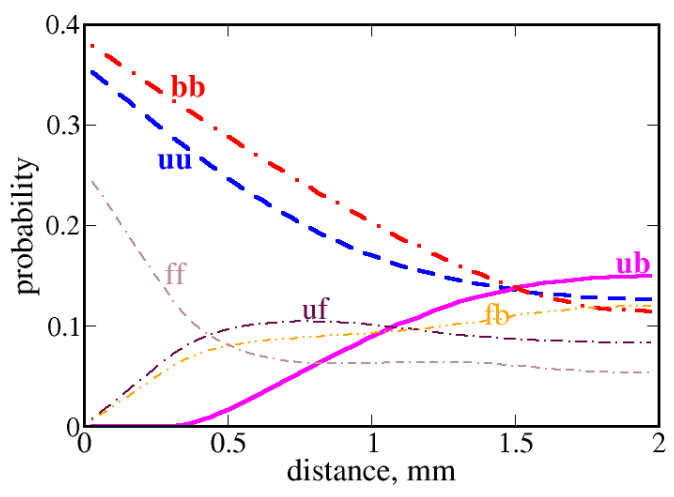

Fig. 2. Probabilities of various events. Legends are explained in caption to Fig. 1.

Third, at the lowest $r \approx r_{0}$ associated with $\mathrm{P}_{12}>0, D_{x x, T, 12} \approx 4.3, D_{y z, T, 12} \approx 0.07$, and $D_{y z, L, 12} \approx 5.0 \mathrm{~m}^{2} / \mathrm{s}^{2}$, respectively, whereas the laminar-flame $(\Delta \mathbf{u})_{L}^{2} \equiv\left[(\sigma-1) S_{L}\right]^{2}=15.4 \mathrm{~m}^{2} / \mathrm{s}^{2}$ in case $\mathrm{H}$. The very small magnitude (when compared to $\left.(\Delta \mathbf{u})_{L}^{2}\right)$ of the transverse unburned-burned $D_{y z, T, 12}$ at $r \approx r_{0}$ also indicates that flamelets associated with the lowest distance $r_{y}$ or $r_{z}$ between the reactants and products are almost parallel to the $z$ or $y$-axis, respectively. Otherwise, both velocities $w$ and $v$ and axes $z$ and $y$ would be partially normal to such a flamelet and, consequently, contributions from $D_{z z, T, 12}$, i.e., difference in $w$ in the $y$-direction, and $D_{y y, T, 12}$, i.e., difference in $v$ in the $z$-direction, would yield a larger $D_{y z, T, 12}$ due to jumps of the locally normal components of $w$ and $v$ across the flamelet. Then, based on the fact that the longitudinal unburned-burned $D_{y z, L, 12}\left(r \approx r_{0}, \bar{c}=0.5\right)$, which is locally (for the considered flamelets, which are parallel to the $y$ or $z$-direction and, hence, normal to the $z$ or $y$-direction, respectively) controlled either by variation of $v$ in the $y$ direction or by variation of $w$ in the $z$-direction, is substantially less than $(\Delta \mathbf{u})_{L}^{2}$, one may conclude that the velocity field in the flamelet is significantly perturbed when compared to the velocity field in the laminar flame. Furthermore, based on the fact that the transverse unburned-burned $D_{x x, T, 12}\left(r \approx r_{0}, \bar{c}=0.5\right)$ is as large as $4.3 \mathrm{~m}^{2} / \mathrm{s}^{2}$ and is sufficiently close to $D_{y z, L, 12}\left(r \approx r_{0}, \bar{c}=0.5\right)$, one may also conclude that there are significant variations in the axial velocity $u$ across the 
considered flamelets, which the velocity $u$ is almost tangential to $\left(\left|n_{x}\right| \ll 1\right.$, as discussed in the previous paragraph). In other words, the magnitude of the locally normal gradient of the locally tangential velocity within flamelets may be comparable with the magnitude of the normal velocity gradient in the laminar flame. Appearance of such relatively strong shear layers (i) puts into question a common practice [4-6] of modeling velocity variations in turbulent flamelets by invoking expressions derived for the laminar flames and (ii) calls for development of a more advanced modeling approach.

Application of target-directed diagnostics, which were developed to further explore the aforementioned trends that had been revealed by analyzing the conditioned structure functions, did show (i) appearance of flamelets almost parallel to the $y$ or $z$-axis and almost normal to two other axes, as well as (ii) appearance of large locally normal (to such flamelets) gradients of locally tangential velocity $u$ [49].

Fourth, an increase in the transverse unburned-burned structure function $D_{x x, T, 12}$ for the axial velocity $u$ with $r$, see solid line (ub) in Fig. 1a, may be attributed to a mechanism highlighted by Scurlock and Grover [2] and by Libby and Bray [50]. If reactant coordinate $\mathbf{x}_{A}$ is kept constant, but the distance $r$ is increased, the axial distance between products in point $\mathrm{B}$ and the local flamelet leading edge is likely to be increased. Therefore, such products are likely to be accelerated by the local axial pressure gradient during a longer time required to reach the more distant point B. Consequently, their axial velocity is higher. A decrease in the longitudinal unburned-burned structure function $D_{y z, L, 12}$ for the transverse velocity $v$ or $w$ with $r$, see solid line (ub) in Fig. 1c, may be attributed to the same mechanism. If the products are accelerated in the axial direction, the magnitude of their transverse velocity decreases due to mass conservation, because the product density is constant.

Fifth, the magnitudes of burned-burned structure functions are typically larger than the magnitudes of unburnedunburned ones, cf. dotted-dashed (bb) and dashed (uu) lines in Fig. 1, because $|\mathbf{u}|$ is higher in the products due to thermal expansion. However, Fig. $1 \mathrm{~b}$ shows the opposite trend for the transverse structure functions $D_{y z, T, 11}(r, \bar{c}=0.5)$ and $D_{y z, T, 22}(r, \bar{c}=0.5)$ for the transverse velocity $v$ or $w$ at $r_{0}<r<0.5 \mathrm{~mm}$, cf. dotted-dashed (bb) and dashed (uu) lines. Such less pronounced small-scale spatial variations in two-point velocity differences in the products when compared to the reactants may be attributed to disappearance of small-scale eddies due to dilatation and increased viscosity. Such effects were documented in numerical [51] and experimental [52] studies of interaction of a laminar premixed flame with a vortex, as well as in recent experiments with turbulent flames [53].

The conditioned structure functions appear to be particularly useful for exploring eventual influence of thermal expansion in premixed flames on the incoming turbulent flow of unburned reactants and such an influence was observed by analyzing the present DNS data. For instance, Fig. 3 shows the longitudinal unburned-unburned structure functions $D_{y z, L, 11}(r, x)$ for the transverse velocity $v$ or $w$ computed in the reactants upstream of and within turbulent flame brushes in cases $\mathrm{H}$ and L. Upstream of the flame brushes, see curves shown in dots and double-dashed-dotted lines, evolution of 
$D_{y z, L, 11}(r, x)$ look similar in both cases. A decrease in $D_{y z, L, 11}(r<1 \mathrm{~mm})$ with $x$, i.e., less-pronounced small-scale spatial variations in the velocity difference at larger $x$, could be attributed to an increase in length scales of the spatially decaying turbulence. However, the behavior of $D_{y z, L, 11}(r, x)$ within flame brushes is different in cases $\mathrm{H}$ and $\mathrm{L}$, cf. curves shown in dotted-dashed or solid lines in Figs. 3a and 3b, respectively. In particular, $D_{y z, L, 11}(r, x)$ at $\bar{c}=0.75$ is largest or lowest in case $\mathrm{H}$ or L, respectively, see solid lines in Figs. 3a and 3b.
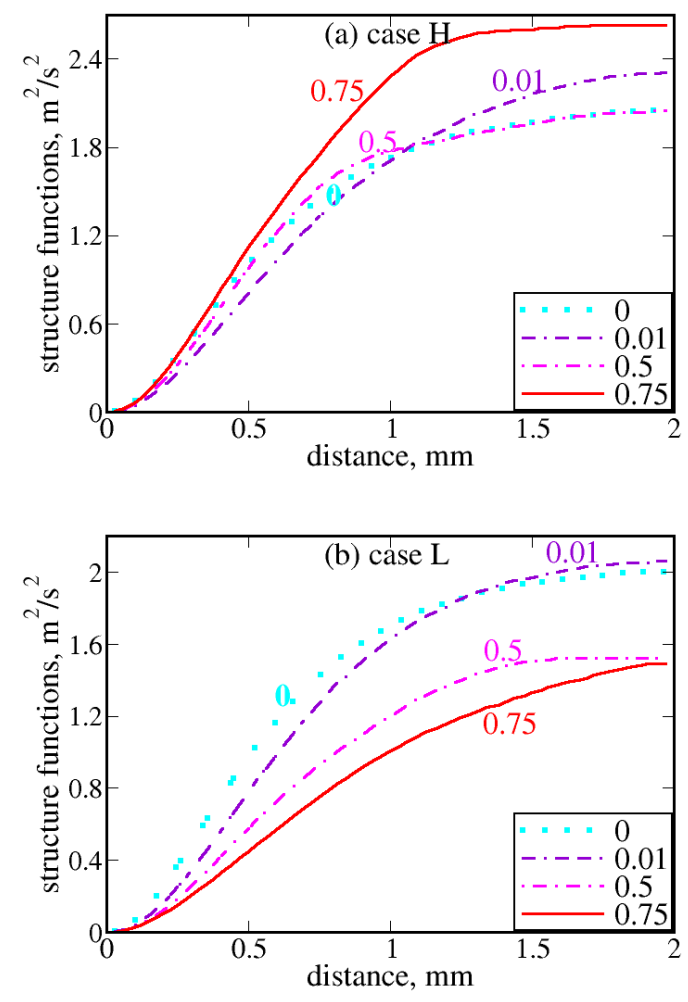

Fig. 3. Longitudinal unburned-unburned structure functions $D_{y z, L, 11}(r, x)$ for the transverse velocity $v$ or $w$ normalized using $\left(v^{\prime 2}+w^{\prime 2}\right) / 2$ conditioned to the reactants and computed at various values of $\bar{c}(x)$, specified in legends. The structure functions marked with 0 and shown in cyan dots were computed at $x=0.25 \mathrm{~mm}$, i.e., well upstream of the flame brushes.

This difference between cases $\mathrm{H}$ and $\mathrm{L}$ is even more pronounced if the considered structure functions $D_{y z, L, 11}(r, \bar{c})$ are "compensated" by dividing them to reference structure functions $D_{y z, L, 11}(r, x=0.25 \mathrm{~mm})$, which characterize the incoming turbulent flow and are almost the same in cases $\mathrm{H}$ and L, see dots in Fig. 3. Figure 4b (case L) shows that $D_{y z, L, 11}(r, x) / D_{y z, L, 11}(r, x=0.25 \mathrm{~mm})$ monotonously decreases with $\bar{c}$ at small scales $(r<1 \mathrm{~mm})$. On the contrary, curves plotted in dotted-dashed and solid lines in Fig. 4a (case $\mathrm{H})$ show that $D_{y z, L, 11}(r, x) / D_{y z, L, 11}(r, x=0.25 \mathrm{~mm})$ increases with increasing $\bar{c}$ provided that $r<1 \mathrm{~mm}$. Moreover, a well pronounced local maximum of $D_{y z, L, 11}(r, \bar{c}=0.5) / D_{y z, L, 11}(r, x=0.25 \mathrm{~mm})$ is observed at $r \approx 0.7 \mathrm{~mm}\left(r \approx 3.5 \delta_{L}\right)$, see dotted-dashed line in Fig. $4 \mathrm{a}$. Furthermore, the influence of the density ratio on $D_{y z, L, 11}(r, \bar{c}) / D_{y z, L, 11}(r, x=0.25 \mathrm{~mm})$ is well pronounced even at 
$\bar{c}=0.25$, cf. curves shown in double-dotted-dashed lines in Figs. $4 \mathrm{a}$ and $4 \mathrm{~b}$. These results indicate that combustion-induced thermal expansion can substantially affect small-scale two-point velocity statistics in the incoming turbulent flow of unburned reactants, thus, casting doubts on relevance of approaches developed for non-reacting turbulent flows, e.g., the Kolmogorov theory, to describing turbulence seen by flamelets within a premixed turbulent flame brush.
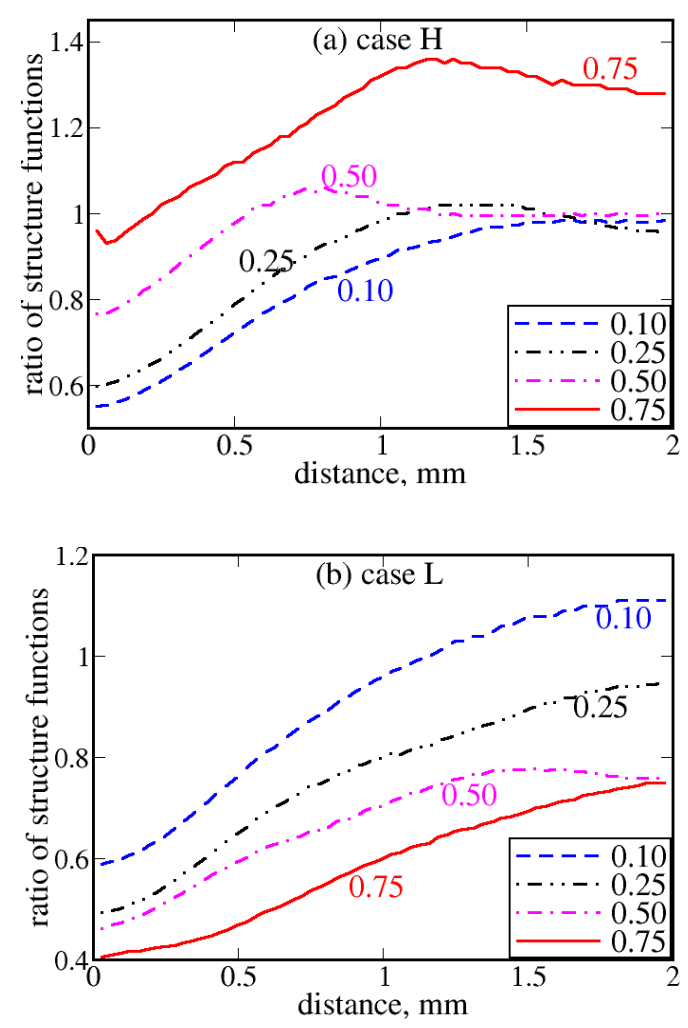

Fig. 4. Ratios of the longitudinal structure function conditioned to reactants $D_{y z, L, 11}(r, x)$ to a reference structure function $D_{y z, L, 11}(r, x=0.25 \mathrm{~mm})$, obtained at various values of $\bar{c}(x)$, specified in legends.

\section{Conclusions}

Conditioned second-order structure functions of velocity field were introduced in order to study the influence of combustion-induced thermal expansion on turbulent flow within a premixed turbulent flame brush. Application of this newly developed method to DNS data obtained from two weakly turbulent premixed flames characterized by significantly different density ratios showed that the structure functions convey information important for understanding fundamentals of flameturbulence interaction and finding issues that require further research. In particular, the reported results indicate that (i) locally normal (to flamelet surface) gradients of locally tangential velocity appear within flamelets and may have significant magnitude (ii) the thermal expansion substantially affects small-scale two-point velocity statistics in the incoming turbulent flow of unburned reactants. The newly introduced conditioned structure-function approach deserves further development and applications using both experiments and simulations. 


\section{Acknowledgement}

VS gratefully acknowledges the financial support provided by ONERA and by the Grant of the Ministry of Education and Science of the Russian Federation (Contract No. 14.G39.31.0001 of 13.02.2017). AL gratefully acknowledges the financial support provided by CERC and Chalmers Area of Advance Transport.

\section{References}

[1] B. Karlovitz, D.W. Denniston, F.E. Wells, J. Chem. Phys. 19 (1951) 541-547.

[2] A.C. Scurlock, J.H. Grover, Proc. Combust. Inst. 4 (1953) 645-658.

[3] R. Günther, Prog. Energy Combust. Sci. 9 (1983) 105-154.

[4] K.N.C. Bray, Proc. R. Soc. London A 451 (1995) 231-256.

[5] A.N. Lipatnikov, J. Chomiak, Prog. Energy Combust. Sci. 36 (2010) 1-102.

[6] V.A. Sabelnikov, A.N. Lipatnikov, Annu. Rev. Fluid Mech. 49 (2017) 91-117.

[7] D.R. Ballal, Proc. R. Soc. London A 367 (1979) 353-380.

[8] I. Gökalp, I.G. Shepherd, R.K. Cheng, Combust. Flame 71 (1988) 313-323.

[9] B.D. Videto, D.A. Santavicca, Combust. Sci. Technol. 70 (1990) 47-73.

[10] J. Furukawa, K. Okamoto, T. Hirano, Proc. Combust. Inst. 26 (1996) 405-412.

[11] J. Furukawa, Y. Noguchi, T. Hirano, Combust. Sci. Technol. 154 (2000) 163-178.

[12] J. Furukawa, Y. Noguchi, T. Hirano, F.A. Williams, J. Fluid Mech. 462 (2002) 209-243.

[13] H. Kolla, E.R. Hawkes, A.R. Kerstein, N. Swaminathan, J.H. Chen, J. Fluid Mech. 754 (2014) 456-487.

[14] A.N. Lipatnikov, J. Chomiak, V.A. Sabelnikov, S. Nishiki, T. Hasegawa, in: M.I. Radulescu (Ed.), Proc. 25th Int. Colloq. Dynamics Explosions Reactive Systems, ICDERS, Leeds, U.K., 2015, paper 74.

[15] C.A.Z. Towery, A.Y. Poludnenko, J. Urzay, J. O'Brien, M. Ihme, P.E. Hamlington, Phys. Rev. E 93 (2016) 053115.

[16] J. O'Brien, C.A.Z. Towery, P.E. Hamlington, M. Ihme, A.Y. Poludnenko, J. Urzay, Proc. Combust. Inst. 36 (2017) 1967-1975.

[17] A.S. Monin, A.M. Yaglom, Statistical Fluid Mechanics: Mechanics of Turbulence, vol. 2, MIT Press, Cambridge, Massachusetts, 1971.

[18] V.R. Kuznetsov, V.A. Sabelnikov, Turbulence and Combustion, Hemisphere Publ. Corp., New York, 1990.

[19] U. Frisch. Turbulence: The Legacy of A.N. Kolmogorov, Cambridge University Press, Cambridge, U.K., 1995.

[20] S.B. Pope, Turbulent Flows, Cambridge University Press, Cambridge, U.K., 2000.

[21] P.A. Davidson, Turbulence: An Introduction for Scientists and Engineers, 2nd ed., Oxford University Press, New York, 2015.

[22] M. Lesieur, O. Metais, P. Comte, Large-Eddy Simulations of Turbulence, Cambridge University Press, Cambridge, U.K., 2005.

[23] A.N. Kolmogorov, Doklady Akademii Nauk SSSR 30 (1941) 301-305.

[24] A.M. Obukhov, Doklady Akademii Nauk SSSR 32(1) (1941) 22-24.

[25] V.R. Kuznetsov, Combust. Explos. Shock Waves 18 (1982) 172-179.

[26] L.D. Landau, E.M. Lifshitz, Fluid Mechanics, Pergamon Press, Oxford, U.K., 1987.

[27] S. Nishiki, T. Hasegawa, R. Borghi, R. Himeno, Proc. Combust. Inst. 29 (2002) 2017-2022.

[28] S. Nishiki, T. Hasegawa, R. Borghi, R. Himeno, Combust. Theory Modelling 10 (2006) 39-55.

[29] H.A. Uranakara, S. Chaudhuri, H.L. Dave, P.G. Arias, H.G. Im, Combust. Flame 163 (2016) 220-240.

[30] A.J. Aspden, M.J. Day, J.B. Bell, Combust. Flame 165 (2016) 266-283.

[31] S. Lapointe, G. Blanquart, Combust. Flame 167 (2016) 294-307.

[32] H. Wang, E.R. Hawkes, B. Zhou, J.H. Chen, Z. Li, M. Aldén, Proc. Combust. Inst. 36 (2017) 2045-2053.

[33] S. Chaudhuri, H. Kolla, H.L. Dave, E.R. Hawkes, J.H. Chen, C.K. Law, Combust. Flame 184 (2017) 273-285.

[34] A.N. Lipatnikov, S. Nishiki, T. Hasegawa, Combust. Theory Modell. 19 (2015) 309-328.

[35] Y.H. Im, K.Y. Huh, S. Nishiki, T. Hasegawa, Combust. Flame 137 (2004) 478-488.

[36] A. Mura, K. Tsuboi, T. Hasegawa, Combust. Theory Modelling 12 (2008) 671-698.

[37] A. Mura, V. Robin, M. Champion, T. Hasegawa, Flow Turbul. Combust. 82 (2009) 339-358.

[38] V. Robin, A. Mura, M. Champion, T. Hasegawa, Combust. Sci. Technol. 182 (2010) 449-464.

[39] V. Robin, A. Mura, M. Champion, J. Fluid Mech. 689 (2011) 149-182.

[40] K.N.C. Bray, M. Champion, P.A. Libby, N. Swaminathan, Combust. Flame 158 (2011) 2017-2022.

[41] A.N. Lipatnikov, S. Nishiki, T. Hasegawa, Phys. Fluids 26 (2014) 105104.

[42] A.N. Lipatnikov, J. Chomiak, V.A. Sabelnikov, S. Nishiki, T. Hasegawa, Proc. Combust. Inst. 35 (2015) 1401-1408.

[43] A.N. Lipatnikov, V.A. Sabelnikov, S. Nishiki, T. Hasegawa, N. Chakraborty, Flow Turbul. Combust. 94 (2015) 513 526.

[44] V.A. Sabelnikov, A.N. Lipatnikov, N. Chakraborty, S. Nishiki, T. Hasegawa, Phys. Fluids 28 (2016) 081701.

[45] A.N. Lipatnikov, V.A. Sabelnikov, S. Nishiki, T. Hasegawa, Combust. Theory Modell. 21 (2017) 205-227. 
[46] V.A. Sabelnikov, A.N. Lipatnikov, N. Chakraborty, S. Nishiki, T. Hasegawa, Proc. Combust. Inst. 36 (2017) 18931901.

[47] A.N. Lipatnikov, V.A. Sabelnikov, N. Chakraborty, S. Nishiki, T. Hasegawa, Flow Turbul. Combust. 100 (2018) 75-92.

[48] A.N. Lipatnikov, J. Chomiak, V.A. Sabelnikov, S. Nishiki, T. Hasegawa, Combust. Theory Modell. 22 (2018) 131-155.

[49] A.N. Lipatnikov, V.A. Sabelnikov, S. Nishiki, T. Hasegawa, Phys. Fluids, submitted.

[50] P.A. Libby, K.N.C. Bray, AIAA J. 19 (1981) 205-213.

[51] T. Poinsot, D. Veynante, S. Candel, J. Fluid Mech. 228 (1991) 561-606.

[52] W.L. Roberts, J.F. Driscoll, M.C. Drake, L.P. Goss, Combust. Flame 94 (1993) 58-69.

[53] T.M. Wabel, A.W. Skiba, J.F. Driscoll, Combust. Flame 188(1) (2018) 13-27. 


\section{Figure captions}

Fig. 1. Conditioned (color lines) and mean (black dots) structure functions obtained at $\bar{c}=0.50$ in case H. (a) Transverse structure functions $D_{x x, T}(r, \bar{c}=0.50)$ for axial velocity. (b) Transverse structure functions $D_{y z, T}(r, \bar{c}=0.50)$ for transverse velocities. (c) Longitudinal structure functions $D_{y z, L}(r, \bar{c}=0.50)$ for transverse velocities. The structure functions are conditioned to the following events; unburned mixture in both points (blue bold dashed lines marked with uu), unburned and burned mixtures (magenta bold solid lines, ub), unburned mixture and flamelet (maroon fine dotted-dashed lines, uf), burned mixture in both points (red bold dotted-dashed lines, bb), burned mixture and flamelet (orange fine double-dotted-dashed lines, fb), and flamelet in both points (brown fine double-dashed-dotted lines, ff).

Fig. 2. Probabilities of various events. Legends are explained in caption to Fig. 1.

Fig. 3. Longitudinal unburned-unburned structure functions $D_{y z, L, 11}(r, x)$ for the transverse velocity $v$ or $w$ normalized using $\left(v^{\prime 2}+w^{\prime 2}\right) / 2$ conditioned to the reactants and computed at various values of $\bar{c}(x)$, specified in legends. The structure functions marked with 0 and shown in cyan dots were computed at $x=0.25 \mathrm{~mm}$, i.e., well upstream of the flame brushes.

Fig. 4. Fig. 4. Ratios of the longitudinal structure function conditioned to reactants $D_{y z, L, 11}(r, x)$ to a reference structure function $D_{y z, L, 11}(r, x=0.25 \mathrm{~mm})$, obtained at various values of $\bar{c}(x)$, specified in legends. 